\title{
Radioactive Iodine Treatment and Cancer Mortality in Hyperthyroid Patients: Questioning Standard Clinical Practice Requires Indisputable Scientific Data
}

\author{
Wengen Chen and Vasken Dilsizian \\ Department of Diagnostic Radiology and Nuclear Medicine, University of Maryland School of Medicine, Baltimore, Maryland
}

$\mathbf{I}_{n}$ quickly (and often without sufficient scrutiny) to the attention of the general public, nuclear medicine physicians are challenged to respond to patients who present with fear and anxiety about potential sequelae to radionuclide therapy. A recent article published in JAMA Internal Medicine (1) suggested that radioactive iodine $\left({ }^{131} \mathrm{I}-\mathrm{NaI}\right)$ treatment is associated with increased cancer mortality in patients with hyperthyroidism. Despite the fact that radioactive iodine has been a mainstay treatment for hyperthyroidism since the 1940s, the article was widely covered in the media and caused alarm among patients considering treatment.

The study, written by Kitahara et al., analyzed solid-cancer mortality in the Cooperative Thyrotoxicosis Therapy Follow-up Cohort Study, which enrolled more than 35,000 patients with hyperthyroidism between 1946 and 1964. Using these data, the authors evaluated the relationship between site-specific cancer death and corresponding organ-absorbed radiation dose among patients who received ${ }^{131} \mathrm{I}$-NaI treatment $(18,805$ of the 35,000 total). A multivariable-adjusted linear dose-response model was applied to calculate excess relative risks per 100-mGy dose to the organs. Excess relative risk was subsequently converted to relative risk and compared in patients with different organ-dose exposure levels.

The results showed that only the breast-and no other organhad a dose-response relationship. Moreover, the dose response for the breast indicated a small relative risk of 1.12 at $100-\mathrm{mGy}$ to the breast $(n=291 ; 95 \%$ confidence interval $[\mathrm{CI}]$ of $1.003-1.32 ; P=$ 0.04). The authors then combined all other solid cancers $(n=$ 1,693 , excluding breast cancer) and found a small but statistically significant dose-response relationship, using stomach-absorbed dose as a surrogate (relative risk at $100-\mathrm{mGy}$ dose to the stomach $=$ $1.05 ; 95 \% \mathrm{CI}$ of $1.01-1.10 ; P=0.01$ ). The authors concluded that radioactive iodine treatment in hyperthyroid patients may be associated with increased cancer mortality. However, the published study immediately raised several concerns about study design and bias-concerns reiterated by some of the study's own authors (2) as well as nuclear medicine physicians outside the United States (3).

Received Aug. 22, 2019; revision accepted Sep. 25, 2019.

For correspondence contact: Wegen Chen, Department of Diagnostic Radiology and Nuclear Medicine, University of Maryland School of Medicine, 22 S. Greene St., Baltimore, MD 21201.

E-mail: wchen5@umm.edu

Published online Oct. 4, 2019.

COPYRIGHT (c) 2019 by the Society of Nuclear Medicine and Molecular Imaging. DOI: 10.2967/jnumed.119.234849

\section{DO METHODOLOGY AND DATA INTERPRETATION SUPPORT THE CONCLUSION?}

The Cooperative Thyrotoxicosis Therapy Cohort Study was not designed to follow up on cancer mortality. Therefore, major confounding factors related to cancer mortality were lacking in the records for this epidemiologic study. For example, the authors presented no data on lifestyles (e.g., alcohol consumption, smoking, or dietary habits), socioeconomic status (e.g., education and income levels), female reproductive history (a critical factor in breast cancer studies), other medical comorbidities, or available medical resources for cancer diagnosis and treatment. The authors did adjust for birth cohort, age, sex, ${ }^{131}$ I-NaI activity, Graves disease, additional surgical procedures, and additional antithyroid drug treatment in the multivariable-adjusted linear dose-response model. Unfortunately, these are not the critical or major confounding factors for cancer mortality. For example, reproductive histories of women (age at menarche and menopause, times of pregnancy and delivery, and lactation) have been consistently shown in epidemiologic studies to be related to breast cancer. Without accounting for these histories in breast cancer mortality, biases will result. This would be similar to implicating lung cancer risks without adjusting for smoking.

It is also unclear why the authors chose the multivariableadjusted linear dose-response model, which analyzed the data of patients who received ${ }^{131} \mathrm{I}-\mathrm{NaI}$ treatment but not those who did not receive the treatment. In the absence of information on confounding factors, an alternate and perhaps preferable approach for analyzing the data would have been to apply multivariate Cox regression for all patients (with and without treatment), where time to cancer death would be the dependent variable and organ-absorbed dose would be the independent variable. The hazard ratio and its 95\% CI would then provide more useful evidence regarding subsequent cancer and associated mortality risk for ${ }^{131} \mathrm{I}-\mathrm{NaI}$ treatment.

\section{HIGH ABSORBED DOSE TO THE ESOPHAGUS BUT NO DOSE-RESPONSE RELATION WITH MORTALITY}

When reviewing the data on organ-absorbed dose estimation, the article indicated that, after the thyroid gland, the esophagus was the organ receiving the second-highest dose (1,600 mGy). This finding was unexpected, because the esophagus does not express the sodium iodine symporter and should not accumulate iodine. No dose-response relationship with mortality was found for the esophagus, which, based on estimations, received a much higher dose than the breast (150 mGy). A lower number of deaths was indicated from esophageal cancer $(n=38)$ than from breast cancer $(n=291)$. 
Only the breast, and no other organs, showed a dose-response relationship. When analyzing all solid tumors combined, the stomach-absorbed dose was used as the surrogate-a puzzling choice, since total-body absorbed dose would have made a more logical surrogate. Relative risk was also small (although statistically significant) for all tumors combined. Statistical significance, of course, is not equivalent to biologic or clinical significance, and ongoing concern has addressed potential overuse and misinterpretation of $P$ values. A recent publication recommended replacing $P$ values with estimates of effects or association and 95\% CIs (4).

Each organ-absorbed dose for an individual patient was calculated using a published biokinetic model, which was previously developed and calibrated using data from a group of 197 hyperthyroidism patients (5). How reliable is the extension of this model to the entire population of 18,805 patients with differing severities of hyperthyroidism? If an organ-dose calculation is not accurate, then any further dose-response analyses could be erroneous or misleading.

Because the population analyzed did not include patients without ${ }^{131} \mathrm{I}-\mathrm{NaI}$ treatment, there should be no data at 0 doses. However, in Figure 1 of the paper, a relative risk of 1 is placed at 0 doses (1). Is this a theoretic value? It is interesting to note that for breast cancer, the relative risks of the 2 highest doses on the right side of the figure are actually located below the estimated log linear dose-response blue line. In a specific example, for the dose at 0.3 , the corresponding relative risk is 1.1 , lower than that of doses at 0.5 and 0.1 . Given the diverse distribution of data, should the reported statistical significance be interpreted as clinically meaningful, particularly when such reports are inevitably delivered without nuance or limitations to the public?

\section{CONCLUSION}

Questioning a time-tested standard clinical treatment for hyperthyroidism requires indisputable scientific data presented in a clear and convincing format and supported by validated and appropriate statistical methodologies. Although we endorse studies that investigate potential sequelae from radioiodine treatment, these should be conducted in well-controlled cohorts. Reporting results with low relative risk from a cohort with bias could deliver confusing or erroneous information, raise unfounded public concern, and adversely affect patient care.

\section{DISCLOSURE}

No potential conflict of interest relevant to this article was reported.

\section{REFERENCES}

1. Kitahara CM, Berrington de Gonzalez A, Bouville A, et al. Association of radioactive iodine treatment with cancer mortality in patients with hyperthyroidism. JAMA Intern Med. July 1, 2019 [Epub ahead of print].

2. Tulchinsky M, Brill AB. Spotlight on the association of radioactive iodine treatment with cancer mortality in patients with hyperthyroidism is keeping the highest risk from antithyroid drugs in the blind spot. Clin Nucl Med. 2019;44: 789-791.

3. Zhang X, Shan G, Liu Q, Lin Y. Regarding the manuscript entitled "Association of radioactive iodine treatment with cancer mortality in patients with hyperthyroidism.” Eur J Nucl Med Mol Imaging. September 6, 2019 [Epub ahead of print].

4. Harrington D, D'Agostino RB Sr, Gatsonis C, et al. New guidelines for statistical reporting in the Journal. N Engl J Med. 2019;381:285-286.

5. Melo DR, Brill AB, Zanzonico P, et al. Organ dose estimates for hyperthyroid patients treated with ${ }^{131} \mathrm{I}$ : an update of the thyrotoxicosis follow-up study. Radiat Res. 2015;184:595-610. 